\title{
Vascular Attributes and Malignant Brain Tumors
}

\author{
Elizabeth Bullitt ${ }^{1}$, Guido Gerig ${ }^{2}$, Stephen Aylward ${ }^{3}$, Sarang Joshi ${ }^{4}$, Keith Smith ${ }^{2}$, \\ Matthew Ewend ${ }^{1}$, and Weili Lin $^{3}$ \\ Departments of ${ }^{1}$ Surgery, ${ }^{2}$ Computer Science, ${ }^{3}$ Radiology, and ${ }^{4}$ Radiation Oncology \\ University of North Carolina, Chapel Hill, NC, 27599, USA \\ \{Bullitt, jksmith, ewend, weili_lin\} @med.unc.edu, \\ \{gerig,joshi\}@cs.unc.edu, aylward@unc.edu
}

\begin{abstract}
Many diseases affect blood vessel morphology. This report analyzes vessel attributes (tortuosity, vessel density, radius, and terminal branch count) within 5 malignant gliomas as seen by high-resolution MR. Results are compared to those in the same anatomical region of 14 normal controls. All tumor patients had marked increases in vessel tortuosity and terminal branch count. These results raise the interesting possibility of automatically defining "vessels of malignancy" within regions of interest on medical images.
\end{abstract}

\section{Introduction}

Blood vessels surround and permeate all organs of the human body. Almost every disease, from cancer to the common cold, affects the vasculature. In this paper, we use the term "vascular attributes" to refer to the combination of "vessel morphology", "vessel density", and "vessel branching pattern". An automated, quantitative calculation of vessel attributes as seen by high resolution, magnetic resonance (MR) images could provide a new and powerful method of diagnosing and staging many diseases.

Malignancy poses a particularly interesting problem. Malignant tumors secrete factors that induce growth of new, abnormally tortuous vessels within the tumor bed. Indeed, in the brain, abnormal vessels provide the most prognostically ominous sign by histological analysis [1], and successful tumor treatment reduces local increases in both vessel number and tortuosity as seen by histological section [2].

The ability to identify and quantitate morphological abnormalities of vessels within MR tumor images would be of high clinical value. Intrinsic brain tumors are graded in terms of increasing malignancy on a scale of I-IV, with treatment dependent upon grade as determined by biopsy. However, each tumor may contain regions of differing malignancy. Moreover, highly malignant tumors, or tumors treated by radiation therapy or other means, may contain necrotic regions in which no tumor cells are present. Sampling error during biopsy thus often underestimates tumor grade, with estimates of false diagnosis in the range of $35 \%-50 \%$ [3]. 
Current imaging attempts to define tumor grade and local regions of malignancy include MR spectroscopy, MR perfusion, and PET scanning. However, none of these methods are yet fully reliable [4], [5], [6], [7]. Disruption of the blood-brain barrier may be one of the confounding factors for perfusion imaging. Vessels are usually not present within necrotic areas, however, and the direct identification of abnormal vessel clusters should be capable of flagging regions of active tumor growth and of high malignancy. Moreover, the ability to measure vessel morphology quantitatively has the exciting potential of defining "vessels of malignancy" and thus of providing a non-invasive means of determining tumor grade. Although this report focuses upon the brain, the same approach is potentially extensible to lesions in any anatomical location, such as the breast or lung.

This paper provides a first exploration of vessel attributes over the population of vessels and vessel segments contained within the margins of known malignant tumors as imaged by MR. For five patients with known high grade gliomas, we compare vessel attributes to those of fourteen normal subjects. For this training set, in which the diagnosis of malignancy was known in advance, we conclude that both tortuosity evaluation and a count of terminal branches appear to be effective when differentiating tumor vessels from those of normal subjects within the same area of interest.

\section{Background}

The concept of providing an automated, quantitative means of measuring vessel attributes is not new. Several groups have proposed analysis of 2D vascular images to predict the likelihood of progressive atherosclerosis or aneurysm formation [8] or to evaluate retinopathy of prematurity [9], [10], [11], [12], [13]. Other groups are working in 3D on the specific problems of carotid stenosis [14] or aortic aneurysms [15]. However, what is needed for widescale utility is analysis of multiple vessel attributes over vessel populations, in 3D, and with the inclusion of small vessels.

One of the most interesting vessel attributes is "tortuosity", or the amount a vessel twists and turns. We have defined three abnormal tortuosity types [16]. Type 1 occurs when a vessel elongates and becomes sinuous. Type 2 is characterized by vessels that make frequent changes of direction and appear as a "can of worms". Type 3 is characterized by high-frequency, low-amplitude oscillations. Tortuosity types 2 and 3 are of greatest relevance to this report since both types appear within malignant tumors.

We have also reported two tortuosity metrics capable of detecting abnormal tortuosity within the intracranial vasculature. The "Inflection Count Metric" (ICM) multiplies the number of inflections present in a 3D space curve by the total path length and divides by the distance between endpoints. The ICM is excellent in defining types 1 and 2 tortuosity, but fails with type 3. The "Sum of Angles Metric" (SOAM) sums curvatures along a sampled curve and normalizes by path length. The SOAM handles tortuosity type 3 well, but can fail with types 1 and 2 [16].

The current report evaluates tortuosity, vessel density, vessel radius, and number of terminal branches (vessels that begin and end within the tumor volume). Given a region of interest demarcated by tumor boundaries, the goal is to seek a set of vascu- 
lar attributes that distinguish the vasculature associated with malignant gliomas from those of normal patients in the same anatomical region. The selection of attributes was based upon what pathologists describe as the characteristics of vascular abnormalities in malignancy — growth of new clusters of abnormally tortuous vessels.

\section{Methods}

Our approach requires segmentation of vessels from MRA and tumors from high resolution MR anatomical images. Coordinate mapping between images is enabled by affine registration of all images with an atlas so that, via a combination of forward and backward transformations, the coordinates of any tumor can be mapped into the undeformed space of any patient's MRA, We report results for five tumors with comparison to the means and standard deviations of vessel attributes within the same anatomical region of fourteen normal subjects.

\subsection{Image Acquisition and Segmentation}

All patients were imaged by 3D, high-resolution, time-of-flight MRA using a quadrature head coil. Inplane resolution was $0.8 \times 0.8 \mathrm{~mm}$ and interslice thickness was $1 \mathrm{~mm}$. All subjects additionally underwent high-resolution T1 and T2 imaging, with tumor patients receiving gadolinium-enhanced T1 studies as well.

Vessel segmentation was done by the method of Aylward [17]. Vessel extraction involves 3 steps: definition of a seed point, automatic extraction of an image intensity ridge representing the vessel's central skeleton, and automatic determination of vessel radius at each skeleton point. The output of the program provides sets of directed, 4dimensional points indicating the $(\mathrm{x}, \mathrm{y}, \mathrm{z})$ spatial position of each sequential vessel skeleton point and an associated radius at each point. Extracted vessels were then postprocessed to produce connected vessel trees and to exclude noise [18].

Tumor segmentation was provided by Gerig. Segmentation in two cases was performed by a new, automated method that uses multi-channel input and an atlas-based approach with five tissue classifiers: grey matter, white matter, CSF, edema, and tumor tissue [19]. Three tumor cases were segmented using a partially manual program that segments tumors via polygon drawing and filling on orthogonal cuts through an image volume. The interactive program is available at http://www.cs.unc.edu/ gerig/. The output of both programs is a mask file in which each voxel associated with object 1 is labeled "1" and voxels unassociated with a segmented object are labeled " 0 ".

\subsection{Image Registration}

The human vasculature is variable. It is thus impossible to provide a one-to-one vascular mapping between patients for more than a few named vessels. Moreover, vessel 
attributes may vary from location to location. Evaluation of disease thus requires knowledge of the mean and variance of normal vessel attributes within a region of interest. Such mapping across patients requires image registration.

For this study, we decided not to transform the vessels, as such transformation might itself alter vessel attributes, but rather to deform the region of interest across patients. Vessels within an area of interest were thus analyzed in their native states. All registrations were performed using Rueckert and Schnabel's mutual informationbased registration program [20], [21], [22]. This program permits rigid, affine, and fully deformable registration. For rigid and affine registrations, the output can be saved as a file convertible to a registration matrix. Settings for rigid and affine registrations included bins $=64$, iterations $=100$, steps $=4$, step length $=2.0$, levels $=3$, and similarity measure=normalized mutual information. For this project, we employed only rigid and full affine registrations and saved the output matrices.

The T1 image of each patient was registered using a full affine registration to the McConnell T1 brain atlas. The additional images of each patient were then rigidly registered to that same patient's T1 image. Lesion coordinates from any tumor patient could then be transformed into the coordinate system of any other patient's MRA via a set of matrix multiplications, with matrix inversion when appropriate. Vessels traversing the region of interest were clipped and analysis was applied only to those vessels and vessel segments lying within the region of interest. This approach therefore calculates vessel attributes only within the undeformed space of each target MRA.

\subsection{Vessel Attributes}

Each tumor patient was compared to fourteen normals. Attributes examined included:

1) Tortuosity as evaluated by both the SOAM and the ICM. Tumor vessels are known to be abnormally tortuous by histological analysis.

2) The ratio of vessel volume to tumor volume. Ingrowth of new vessels might increase the proportion of the volume of interest occupied by vessels.

3) The average radius of the vessels or vessel segments within the region of interest. New vessel growth might produce feeding vessels of larger diameter.

4) Count of terminal branches (branches that begin and end within the volume). As tumors induce vessel ingrowth, an additional number of vessels might be expected.

In some cases, one or more normal patients had no vessels in the anatomical region corresponding to the tumor. In these cases we set the vessel volume and the number of terminal branches to 0 and used the full number of normal patients in the analysis. However, there is no good way to provide a tortuosity value or an average radius if no vessels are present. We therefore evaluated these parameters using only the subset of normal subjects that possessed vessels within the anatomical region of interest.

For each variable, results were averaged to provide a single value for all vessels in a given patient. "Radius", for example, represents the average radius of all vessel points within the volume of interest for a given patient.

Malignant gliomas may encase normal vessels as well as induce growth of new vessels. Whether encased vessels themselves become abnormal is unknown. For this 
initial analysis, we did not attempt to subdivide the vessels of tumor patients into different groups. Results reported for tumor patients are therefore likely to incorporate values produced by encased "normal" vessels as well as results produced by new vascular growth induced by the tumor.

\section{Results}

Figure 1 illustrates a patient with a malignant glioma. The tumor is volume rendered at lower left and is shown registered with segmented, surface-rendered vessels. Note that there are both avascular areas and areas of vessel clustering within the tumor. Segmented vessels are color-coded by relationship to the tumor surface. Dark grey vessels are outside the tumor, light grey vessels traverse the tumor, and medium grey vessels are contained within the tumor. Both traversing and intrinsic tumor vessels have high frequency, low amplitude "wiggles" rarely found outside of a tumor or in normal patients (this abnormality is tortuosity type 3 ). The medium grey vessels also produce a "can of worms" configuration and provide an example of tortuosity type 2 . The medium grey vessels additionally represent an abnormal vessel cluster unlikely to be present within the same anatomical region of normal patients.

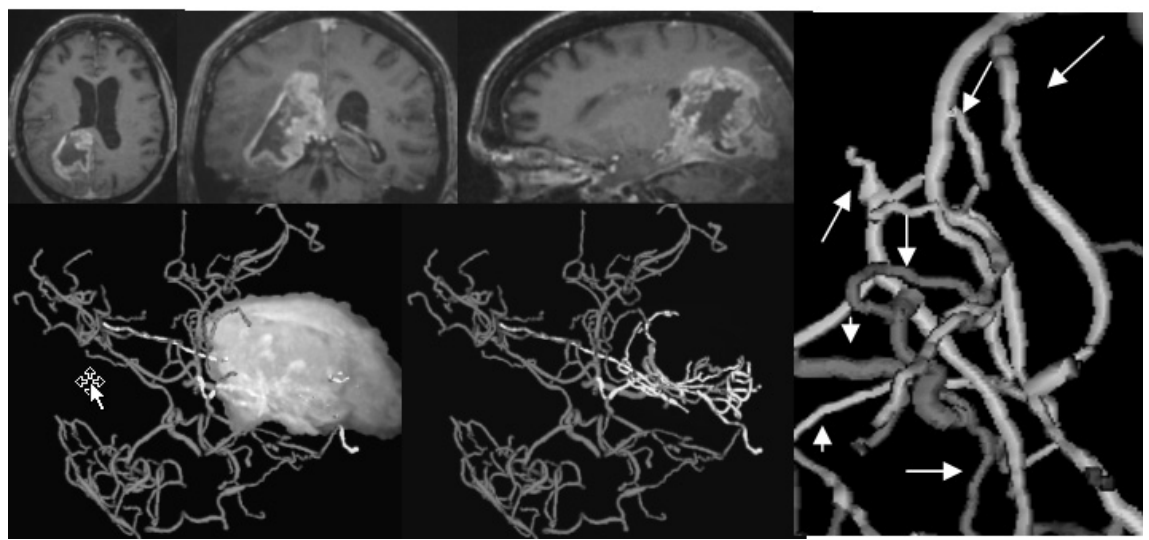

Fig. 1. Malignant glioma. Upper row $=\mathrm{Gad}+\mathrm{MR}$ slices. At bottom left, the tumor is volume rendered at full opacity and shown with surface rendered, segmented vessels. At bottom center, the tumor is set to 0 opacity in order to display the vasculature inside it. The image at far right illustrates foci of abnormal tortuosity type III (arrows) involving the vessels contained within (medium grey) and passing through (light grey) the tumor.

Table 1 shows results for the five tumor cases for all parameters examined. The mean and standard deviation for the fourteen normal controls are given immediately below each tumor value. One star indicates a tumor value more than one standard deviation from normal and three stars indicates a tumor value more than three standard deviations from normal. For this small number of cases, we have focused only 
upon those variables in which all five tumor patients produced values lying more than one standard deviation beyond normal.

As shown by Table 1, some of the variables appear helpful whereas others do not. The first two rows of Table 1 indicate tortuosity evaluation by two metrics. All five tumors exhibited significant increases in tortuosity by both metrics. The volume ratio was less helpful, however. Although three tumors displayed an increase in the proportion of volume occupied by vessels, two did not. The average vessel radius calculation was also not helpful, with some tumors exhibiting a higher average vessel radius and some a lower. On the other hand, the count of terminal branches was markedly different between tumor and normal patients, and all five tumor cases displayed an increased number of terminal branches well more than three standard deviations from normal.

Table 1. Vessel attributes. Results for each tumor are given in a column, with mean and standard deviation of normal immediately below each value. The top row provides the tumor IDs. The leftmost column identifies the parameter analyzed in each row. SOA = In-plane sum-ofangles metric. $\mathrm{IC}=$ inflection count metric. $\mathrm{VR}=$ proportion of the region of interest occupied by vessels (result multiplied by 10 to fit the table). $\mathrm{RAD}=$ average radius of vessels within the region $(\mathrm{cm}) . \mathrm{TB}=$ count of branches fully contained within the region of interest.

\begin{tabular}{cccccc} 
& 024 & 025 & 026 & 031 & 032 \\
\hline SOA & $4.7 * *$ & $4.4^{*}$ & $7.5^{* *}$ & $4.5^{* * *}$ & $6.4^{* * *}$ \\
& $2.8 \pm 0.8$ & $3.5 \pm 0.7$ & $3.9 \pm 2.1$ & $3.1 \pm 0.4$ & $2.6 \pm 0.5$ \\
\hline IC & $20.2 * * *$ & $22.2^{*}$ & $23.8^{* * *}$ & $71.7 *$ & $62.9 * * *$ \\
& $4.3 \pm 2.6$ & $12.9 \pm 6.0$ & $2.3 \pm 1.1$ & $47.2 \pm 15.9$ & $27.3 \pm 7.7$ \\
\hline VR & $0.68^{* * *}$ & $0.09 * *$ & $0.09 * * *$ & 0.05 & 0.07 \\
& $0.05 \pm 0.01$ & $0.04 \pm 0.02$ & $0.01 \pm 0.001$ & $0.05 \pm 0.01$ & $0.06 \pm 0.02$ \\
\hline RAD & $0.11 *$ & $0.09 *$ & $0.05^{*}$ & $0.05^{*}$ & 0.08 \\
& $0.08 \pm 0.02$ & $0.07 \pm 0.01$ & $0.07 \pm 0.01$ & $0.07 \pm 0.01$ & $0.07 \pm 0.01$ \\
\hline TB & $5 * * *$ & $7 * * *$ & $13^{* * *}$ & $16^{* * *}$ & $24 * * *$ \\
& $0.1 \pm 0.5$ & $0.9 \pm 1.2$ & $0.1 \pm 0.3$ & $5.9 \pm 3.2$ & $4.8 \pm 2.5$ \\
\hline
\end{tabular}

\section{Discussion}

This report describes a search for quantifiable differences between the vasculature of normal subjects and malignant glioma patients as seen by high-resolution MRA. We conclude that both tortuosity analysis and terminal branch count appear to be useful discriminative methods. Several points should be made about methods, however.

First, there are multitudinous ways in which one vessel population can be compared to another. It is difficult to know how best to phrase any analysis so as to best quantitatively define differences between groups. This study made a set of guesses based upon qualitative diagnostic criteria used to define malignant gliomas by histological examination. Several criteria that we expected to show significant differences between groups were successful but others were not. In retrospect, some of the 
questions might have been better posed. The analysis of vessel density, for example, calculated the ratio of total vessel volume to total tumor volume. In fact, and as shown by Figure 1, almost all malignant gliomas exhibit both foci of high vascularity and foci of avascularity. A stronger approach might seek for localized regions of vascular clustering and abnormality, and then flag and count these voxels. Such approach would provide a better comparative analysis and could tell the surgeon on a voxel by voxel basis which regions of the tumor appear best for biopsy.

Second, the optimal method of registration is unknown when analyzing vasculature. The methods employed here did not deform the vessels themselves but rather used an affine registration to deform the region of interest across patients. This may or may not prove to be the optimal solution. The development of methods of vascular registration is an active area of research [23].

Third, the vessels of interest are small. High resolution MR (1x1x1 mm) is likely to be required, as is a method of vessel extraction capable of defining small vessels.

Finally, the aim of this study is to define parameters likely to be important in characterizing the vasculature of malignant tumors. Confirmation of the conclusions will require both a larger normal database and testing against a large set of patients bearing tumors of different grades. Moreover, even if the results are confirmed, we do not know what the vascular patterns of metastatic tumors or of lower grade gliomas might be. We do not know if there is a single pattern that characterizes "vessels of malignancy", if a single pattern exists that shows progressive deviation from normal with increasing malignancy, or if multiple, tumor-specific patterns exist. We view all of these questions as enormously exciting areas of research, each of which could be of clinical value.

In summary, this report provides a first exploration of the ability to automatically differentiate, localize, and quantitate populations of abnormal vessels within malignant tumors as seen by medical imaging data. Such ability could have great clinical potential. The initial results are exciting and suggest that, at minimum, malignant tumor vessels can be differentiated from those of normal patients within a similar anatomical region on the basis of both tortuosity and count of terminal branches.

Acknowledgments. This work was supported, in part, by R01 EB000219 NIBIB and R01 HL69808 NIH-HLB. Portions of the software are licensed to Medtronic Corp (Minn., Minn) and R2 Technologies (Los Altos, CA). We are grateful to Daniel Rueckert for providing his registration software and to Stephen Pizer for his advice.

\section{References}

1. Burger PC, Scheithauer BW, Vogel FS.: Surgical Pathology of the Nervous System and its Coverings, Third Edition, Churchill Livingstone, New York (1991)

2. Jain RK : Normalizing tumor vasculature with anti-angiogenic therapy: a new paradigm for combination therapy Nature Medicine 7 (2001) 987-98.

3. Kaufman HH, Ostrow PT, Butler IJ : Diagnostic brain biopsy. In: Wilkins RH, Rengachery SS (eds.) Neurosurgery, McGraw-Hill, New York (1985) 289-294. 
4. Kahn D, Follett KA, Bushnell DL, et al.: Diagnosis of recurrent brain tumor: value of ${ }^{201}$ Tl SPECT vs ${ }^{18}$ F-fluorodeoxyglucose PET. AJR Am J Roentgenol. 1994; 163: 14591465.

5. Yoshii Y, Moritake T, Suzuki K, et al.: Cerebral radiation necrosis with accumulation of thallium 201 on single-photon emission CT. AJNR Am J Neuroradiol. 1996; 17: 17731776.

6. Ricci P.: Differentiating recurrent tumor from radiation necrosis with 18FDG-PET: time for reevaluation? In: Proceedings of the 34th Annual Meeting of the American Society of Neuroradiology. Seattle, Wash; 1996.

7. Benard F, Romsa J, Hustinx R: Imaging gliomas with positron emission tomography and single-photon emission computed tomography. Seminars Nuc. Med. 23 (2003) 148-162.

8. Smedby O, Hogman N, Nilsson S, Erikson U, Olsson AG, Walldius G : Twodimensional tortuosity of the superficial femoral artery in early atherosclerosis. J Vascular Research 30 (1993) 181-191.

9. Bracher D : Changes in peripapillary tortuosity of the central retinal arteries in newborns. Graefe's Arch Clin Exp Opthalmol 218 (1982) 211-217.

10. Zhou LA, Rzeszotarski MS, Singerman LJ, Chokreff JM : The detection and quantification of retinopathy using digital angiograms. IEEE-TMI 13 (1994). 619-626.

11. Goldbaum MH, Hart WE, Cote BL, Raphaelian PV :Automated measures of retinal blood vessel tortuosity. Invest Opthalmol Vis Sci 35 (1994) 2089.

12. Hart WE, Goldbaum M, Cote B, Kube P, Nelson MR Measurement and Classification of Retinal Vascular Tortuosity. Intl J Medical Informatics 53(2-3) (1999). 239-252.

13. Capowski JJ, Kylstra JA, Freedman SF :A numeric index based on spatial frequency for the tortuosity of retinal vessels and its application to plus disease in retinopathy of prematurity. Retina 15 (1995) 490-500.

14. Frangi AF, Niessen WJ, Hoogeveen RM, Walsum TV, Viergever MA : Quantification of vessel morphology from 3D MRA. MICCAI'99 Lecture Notes in Computer Science 1679 (1999) 358-367.

15. De Bruijne M, van Ginneken B, Niessen WJ, Maintz, JBA, Viergever : Active shape model based segmentation of abdominal aortic aneurysms in CTA images. SPIE (2002) 4684: 463-474.

16. Bullitt E, Gerig G, Pizer SM, Lin W, Aylward SR Measuring tortuosity of the intracerebral vasculature from MRA images. Accepted IEEE-TMI pending minor revision; Available at: http://CASILab.med.unc.edu.

17. Aylward S, Bullitt $\mathrm{E}$ : Initialization, noise, singularities and scale in height ridge traversal for tubular object centerline extraction. IEEE-TMI 21 (2002) 61-75.

18. Bullitt E, Aylward S, Smith K, Mukherji S, Jiroutek M, Muller K : Symbolic Description of Intracerebral Vessels Segmented from MRA and Evaluation by Comparison with XRay Angiograms. Medical Image Analysis 5 (2001) 157-169,

19. Prastawa M, Bullitt E, Gerig G (2003) Robust estimation for brain tumor segmentation. Accepted MICCAI 2003.

20. Schnabel JA, Rueckert D, Quist M, Blackall JM, Castellano Smith AD, Hartkens T, Penney GP, Hall WA, Liu H, Truwit CL, Gerritsen FA, Hill DLG, and Hawkes JD: A generic framework for non-rigid registration based on non-uniform multi-level free-form deformations. MICCAI 2001; Lecture Notes in Computer Science 2208 (2001) pp. 573 581.

21. Rueckert D, Sonoda LI, Hayes C, Hill DLG, Leach MO, and Hawkes DJ Non-rigid registration using free-form deformations: Application to breast MR images. IEEE Transactions on Medical Imaging, 18 (1999) 712-721. 
22. Rueckert D (2002) "Rview". Available: www.doc.ic.ac.uk/ dr/software.

23. Aylward SR, Jomier J, Weeks S, and Bullitt E: Registration and analysis of vascular images. In press IJCV. 